\title{
The effect of prenatal education curriculum on mother's prenatal examination utilization, delivery mode and recovery status: a cross-sectional survey in China
}

\author{
Yuhui Shi $^{1}$ - Dongxu Wang ${ }^{2}$ Yanfei Yuan ${ }^{3}$ Ying Jiang' ${ }^{1}$ Qingqi Zeng ${ }^{1}$. \\ Chun Chang ${ }^{1}$
}

Received: 7 April 2015/Accepted: 3 July 2015/Published online: 23 July 2015

(C) The Japanese Society for Hygiene 2015

\begin{abstract}
Objectives To examine the participation, implementation, and effect of the prenatal education curriculum provided by hospitals in China, and to provide evidence for the improvement of prenatal education.

Methods A cross-sectional survey was conducted in the hospitals in Hunan Province, China. Mothers aged 20-45 years who had given birth between 1 May 2011 and 1 May 2012 and not diagnosed with pregnancy-related complications were invited to participate in the study. A self-administered, structured questionnaire was used to examine the effect of prenatal education curriculum on prenatal examination utilization, delivery mode, and recovery status from delivery.

Results Among the total 604 respondents, only 175 $(29.1 \%)$ surveyed mothers participated in prenatal education curriculum provided by hospitals during their latest delivery. These mothers had a higher rate of attending all the required prenatal examinations (57.9 vs. $48.3 \%$ ), and a higher rate of recovering very well and well ( 80 vs. $73.7 \%$ ) from the latest delivery, than those who did not participate in prenatal education curriculum $(P<0.05)$. However, there was no statistical difference in the delivery
\end{abstract}

Y. Shi and D. Wang are the co-first authors.

Chun Chang

changchun@bjmu.edu.cn

1 Department of Social Medicine and Health Education, School of Public Health, Peking University, Beijing 100191, China

2 Research School of Population Health, Australian National University, Canberra, ACT 2601, Australia

3 Center for Policy Research and Health Communication, Chinese Center for Disease Control and Prevention, Beijing 102206, China mode between mothers who participated and those who did not participate in the prenatal education curriculum provided by hospitals.

Conclusions Prenatal education is indispensable for the improvement of maternal and child health, and thus should be advocated. In China, a standard and convenient specification prenatal education curriculum provided by hospitals and their doctors is appropriated for providing prenatal education to pregnant women.

Keywords Effect - Prenatal education - Curriculum . China

\section{Introduction}

Pregnancy, labor, and postpartum period constitute an important life experience for women. The process of creating a new human being calls on a woman to learn new information about her body and to invest in her health and the health of her growing fetus [1]. Ensuring that pregnant women have universal and timely access to quality prenatal care is a global public health priority, because it is an important determinant of improved health outcomes of infants and mothers [2]. Prenatal care offers multiple opportunities to reach expectant mothers with information on any risks related to labor and delivery, and as a way of promoting delivery with the assistance of a skilled healthcare provider [3]. Timely and adequate prenatal care has also been found to be important for the health of newborns [4]. In addition, prenatal care can be an entry point for the prevention of HIV transmission from mother to children. Prenatal care attendance can also, especially in rural settings, facilitate women's access to medical care for future needs [5]. 
Prenatal education that takes place as a part of routine prenatal care has long been identified as an important component of prenatal care [6]. Prenatal education can increase awareness and improve knowledge about risk factors and complications of pregnant women. The goals of prenatal education are to help parents become knowledgeable mothers and fathers, make them take an active role in maintaining health during pregnancy and birth and teach coping techniques to deal with pregnancy, childbirth and parenting [7]. Therefore, prenatal education is important in improving health of infants and mothers, and should be implemented widely [8].

In China, the National Health and Family Planning Commission (NHFPC) has recommended hospitals to provide separate prenatal education curriculum to pregnant women; however, there is no required universal standard and protocol on the delivery of the prenatal education curriculum. Additionally, there are no studies examining the effect of the prenatal education curriculum. However, it is well acknowledged that a comprehensive assessment of prenatal education curriculum and its effect is a critical step in the development of effective prenatal education programs, which have a positive influence on the improved health of mothers and infants accordingly. In view of this, the objectives of this study are to examine the participation, implementation, and effect of the current prenatal education curriculum provided by hospitals in China, and to provide evidence for the improvement of prenatal education in the future.

\section{Materials and methods}

The study received ethical clearance approval from the Peking University Institutional Review Board (Ethics Review Approval No: IRB00001052-12034), approved on 28 June 2012. Before starting the survey, each participant was provided with full information about the study, and informed consent signed by each participant was obtained in accordance with the ethical standards of the Helsinki Declaration.

\section{Study design}

A cross-sectional survey was conducted from July to September 2012 in Hunan Province, China. A two-staged cluster sampling method was used to enroll the participants. Hospitals were divided into provincial, city and county levels and two hospitals were randomly selected from each level. The following equation was used to calculate sample size in each cluster: $n=\operatorname{deff} \times \mu_{a}^{2} \pi(1-\pi) / \delta^{2}$, where $\mu_{a}$ was given a value of $1.96, \delta$ was given a value of 0.1 and deff was given a value of 2 . According to a survey conducted by health department of Hunan province in 2011, the average CS rate in Hunan was $53.20 \%$ [9], thus $\pi$ was given a value of 0.532 . Considering a loss rate of $5 \%$, we increased the sample size to 202 in each cluster. Mothers aged 20-45 years whom had given birth (by natural delivery or cesarean section) between 1 May 2011 and 1 May 2012 in these hospitals were invited to participate in our study. Those diagnosed with pregnancy-related complications, such as pregnancy-induced hypertension, gestational diabetes, etc., were excluded from our research in order to remove pregnancy-related complications' influence on C-section selection intention of the participants. The participation, implementation and effect of prenatal education curriculum were measured by using a questionnaire.

\section{Measures}

The instrument for the survey was a 26 -item, self-administered, structured questionnaire, designed in Chinese. The questionnaire was divided into five main sections to assess respondents' personal information, their prenatal examination utilization, delivery mode, and recovery status from the delivery, and the participation, implementation and effect of the prenatal education curriculum. A consistency test was used to evaluate the main section (prenatal education curriculum) of the questionnaire, the mean Cronbach's alpha coefficient was 0.825, indicating good consistency of the prenatal education curriculum section, and thus the questionnaire can be used in the investigation.

To be specific, in Section A (personal information) mothers' and their husband's age, educational level, occupation, and monthly income were asked. In Section B (prenatal examination utilization), mothers were asked "did you attend the prenatal examination as required during your latest delivery?" (based on the requirements in the National Basic Public Health Service Specification 2011 issued by the Chinese NHFPC [10]). In Section C (delivery mode), mothers were asked "what was your latest delivery mode?" In Section D (recovery status), mothers were asked "what do you think about your recovery status from the latest delivery?" In Section E (prenatal education curriculum), mothers were asked "did you participate in prenatal education curriculum during your latest pregnancy?", "what was the most important reason why you participated or did not participate in it?", "what information did the prenatal education curriculum provide for you?", "how was the prenatal education curriculum conducted?", and "what do you think about the effect of the prenatal education curriculum?"

\section{Data collection}

The structured questionnaire was administered from July to September 2012 in Hunan Province after investigators trained by a professor from the School of Public Health, Peking University. Mothers of infants were collected from 
2 kinds of site, one was postpartum clinics, and another was pediatric clinics, where their babies received a health examination 42 days after birth or routine physical examination according to the requirements in the National Basic Public Health Service Specification 2011 issued by the Chinese NHFPC [10]. After introducing the study to the mothers, informed consent was gained from each participant, and the participant was asked to complete an anonymous self-administered questionnaire. Each questionnaire took about $20 \mathrm{~min}$ to complete. Investigators checked each questionnaire for logistic errors and blanks, and any errors and blanks were corrected on-site. Totally 609 mothers participated the survey, and 604 mothers completed the questionnaire as required. A valid response rate was $99.2 \%$.

\section{Statistical analysis}

Using Epidata3.02 software, data were coded and entered onto a computer, and then logical error detection and verification were carried out to exclude missing data and abnormal values. We conducted all of the statistical analysis procedures using the Statistical Package for Social Science version 20.0 (SPSS 20.0). We used an alpha level of 0.05 to determine the statistical significance of the data. Mean and standard deviation were used to summarize mothers' and their husbands' ages. Frequencies were calculated to describe mothers' and their husbands' personal information (education level, occupation, monthly income), the participation, implementation and effect of prenatal education curriculum, prenatal examination utilization, delivery mode and recovery status from the latest delivery. Chi-squared tests were used to identify whether prenatal education curriculum had an influence on mother's prenatal examination utilization, delivery mode, and recovery status from the latest delivery.

\section{Results}

\section{Personal information of mothers and their husbands}

The personal information of the study population was presented in Table 1. Altogether there were 604 mothers who completed the questionnaire, with average age of $(27.6 \pm 4.2)$ years, and average husband's age of $(30.4 \pm 4.7)$ years. $76 \%$ of surveyed mothers graduated from high school, college or university, and this rate was $78.7 \%$ among their husbands. The education levels among mothers who participated in prenatal education curriculum or not had statistically significant difference, also among their husbands $(P<0.05)$. The unemployment rate of surveyed mothers and their husbands was 18.9 and $4.9 \%$, respectively. $24.4 \%$ of surveyed mothers earned more than 3500 RMB per month, while this rate was $51.9 \%$ among their husbands. The differences among monthly income of their husbands were statistically significant $(P<0.05)$.

\section{Participation, implementation and effect of prenatal education curriculum}

From the total respondents, only 175 mothers of infants $(29.1 \%)$ participated in prenatal education curriculum provided by hospitals during their latest delivery. The most important reason why they participated was because they wanted to learn more about health care knowledge during pregnancy $(51.2 \%)$, followed by being advised by their doctor to do so (44.2\%). For the 426 mothers who did not participate in prenatal education curriculum, the most important reason was that they had no time $(41.8 \%)$, followed by they did not have prenatal education curriculum in their hospitals or did not know their hospitals provided the curriculum $(35.6 \%)$.

Table 2 displays the implementation and effect of prenatal education curriculum among mothers. For the formats of prenatal education curriculum, the most common was a lecture $(92.6 \%)$, followed by promotional materials $(64.6 \%)$, video $(60.0 \%)$, demonstration and practice $(58.3 \%)$, and group discussion $(54.3 \%)$. With regard to the effect of the prenatal education curriculum provided by the hospitals, the majority of surveyed mothers who participated in it said they knew more about prenatal examination $(94.3 \%)$, health care knowledge during pregnancy $(90.3 \%)$, roles of doctors and nurses during delivery $(81.1 \%)$, benefits and approaches of breastfeeding $(92.6 \%)$. They further indicated they knew more about how to recover well after delivery $(84.0 \%)$, they felt more trust in hospitals $(82.9 \%)$ and doctors $(84.6 \%)$, they had more confidence in natural delivery $(86.9 \%)$ and less fear of delivery $(86.3 \%)$, and they could communicate better with doctors $(84.6 \%)$. For most of the information, the forms and the effect of prenatal education curriculum had no statistical difference in different level hospitals.

\section{Influence of prenatal education curriculum on mother's prenatal examination utilization, delivery mode and recovery status from the latest delivery}

The influence of prenatal education curriculum on mother's prenatal examination utilization, delivery mode and recovery status from the latest delivery is shown in Table 3. The surveyed mothers who participated in prenatal education curriculum had a higher rate of attending all the required prenatal examinations (57.9 vs. $48.3 \%$ ) and a lower rate of not attending the prenatal examination 
Table 1 Personal information of mothers of infants who participated in prenatal education curriculum or not and their husbands $(n=604)$

\begin{tabular}{|c|c|c|c|c|c|c|}
\hline & \multicolumn{2}{|l|}{ Mothers } & \multirow[t]{2}{*}{$t / \chi^{2}$} & \multicolumn{3}{|l|}{ Their husbands } \\
\hline & $\begin{array}{l}\text { Participated } \\
n(\%)\end{array}$ & Not $n(\%)$ & & $\begin{array}{l}\text { Mother participated } \\
n(\%)\end{array}$ & $\begin{array}{l}\text { Mother not participated } \\
n(\%)\end{array}$ & $t / \chi^{2}$ \\
\hline \multicolumn{7}{|l|}{ Age (years) } \\
\hline Min & 20 & 18 & & 21 & 20 & \\
\hline Max & 42 & 42 & & 46 & 48 & \\
\hline Mean \pm STD & $28.0 \pm 4.1$ & $27.3 \pm 4.2$ & 1.76 & $30.8 \pm 4.7$ & $30.3 \pm 4.8$ & 1.16 \\
\hline \multicolumn{7}{|l|}{ Education } \\
\hline Primary school and below & $1(0.6)$ & $7(1.6)$ & & $1(0.6)$ & $5(1.2)$ & \\
\hline Middle school & $28(16.3)$ & $108(25.4)$ & & $25(14.5)$ & $97(22.8)$ & \\
\hline High school & $42(24.4)$ & $113(26.6)$ & & $34(19.8)$ & $113(26.6)$ & \\
\hline College & $45(26.2)$ & $87(20.5)$ & & $45(26.2)$ & $77(18.1)$ & \\
\hline University & $56(32.6)$ & $110(25.9)$ & $9.5^{*}$ & $67(39.0)$ & $133(31.3)$ & $12.88^{*}$ \\
\hline \multicolumn{7}{|l|}{ Occupation } \\
\hline Staff in farming, forestry, or fishery & $16(9.2)$ & $27(6.4)$ & & $11(6.4)$ & $33(8.0)$ & \\
\hline Staff in manufacture, or transportation & $4(2.3)$ & $6(1.4)$ & & $18(10.5)$ & $40(9.7)$ & \\
\hline Staff in business, or service industry & $28(16.1)$ & $65(15.4)$ & & $32(18.7)$ & $97(23.5)$ & \\
\hline $\begin{array}{l}\text { Manager in government, public institutions or } \\
\text { enterprise }\end{array}$ & $40(22.9)$ & $81(19.3)$ & & $44(25.7)$ & $80(19.4)$ & \\
\hline Professionals & $33(19.0)$ & $53(12.6)$ & & $48(28.1)$ & $108(26.2)$ & \\
\hline Soldiers & - & $1(0.2)$ & & $2(1.2)$ & $7(1.7)$ & \\
\hline Housework & $25(14.4)$ & $86(20.4)$ & & $3(1.8)$ & $9(2.2)$ & \\
\hline Unemployed & $22(12.6)$ & $91(21.6)$ & & $5(2.9)$ & $24(5.8)$ & \\
\hline Others & $6(3.4)$ & $11(2.6)$ & 15.16 & $8(4.7)$ & $15(3.6)$ & 9.09 \\
\hline \multicolumn{7}{|l|}{ Monthly income (RMB) } \\
\hline 199 and below & $29(17.3)$ & $101(24.1)$ & & - & $8(1.9)$ & \\
\hline $200-1019$ & $22(13.1)$ & $57(13.6)$ & & $5(2.9)$ & $31(7.4)$ & \\
\hline 1020-3499 & $66(39.3)$ & $168(40.1)$ & & $65(38.0)$ & 174 (41.6) & \\
\hline $3500-5999$ & $36(21.4)$ & $64(15.3)$ & & $67(39.2)$ & $131(31.3)$ & \\
\hline 6000-8499 & $10(6.0)$ & $17(4.1)$ & & $24(14.0)$ & $38(9.1)$ & \\
\hline 8500 and above & $5(3.0)$ & $12(2.9)$ & 6.17 & $10(5.8)$ & $36(8.6)$ & $13.90 *$ \\
\hline
\end{tabular}

$* P<0.05$

at all ( 0 vs. $0.5 \%)$, than those who did not participate in prenatal education curriculum. There was a significant difference in mothers' prenatal examination utilization between those who participated and those who did not participate in prenatal education curriculum $(P<0.05)$. Mothers who participated in prenatal education curriculum had a higher rate of recovering very well and well (80 vs. $73.7 \%$ ) and a lower rate of recovering not well (1.1 vs. $2.8 \%$ ) from the latest delivery, than those who did not participate in prenatal education curriculum. There was a significant difference in mothers' recovery status from the latest delivery between those who participated and those who did not participate in prenatal education curriculum $(P<0.05)$. The rate of natural delivery among mothers who participated in prenatal education curriculum was lower than those who did not participate in prenatal education curriculum (44.0 vs. $46.6 \%$ ); however, there was no significant difference in mothers' latest delivery mode between those who participated and those who did not participate in prenatal education curriculum $(P<0.05)$.

\section{Discussion}

In this study, we found that the participation rate of prenatal education curriculum was very low, and the most important reasons for this low rate were lack of time or access to the curriculum in their hospitals, or not knowing that hospitals provided this curriculum. For those who had participated in the prenatal education curriculum, their willingness to learn more knowledge about health care during pregnancy and their doctor's advice were the key reasons for their participation. It was found that the prenatal education curriculum provided a wide range of information for pregnant women 
Table 2 Implementation and effect of prenatal education curriculum among mothers of infants in different level hospitals $(n=175)$

\begin{tabular}{|c|c|c|c|c|c|}
\hline Content & Province $n(\%)$ & City $n(\%)$ & County $n(\%)$ & Total $n(\%)$ & $P^{*}$ \\
\hline \multicolumn{6}{|c|}{ Information provided by prenatal education curriculum you participated } \\
\hline Prenatal examination and neonatal screening & $41(63.1)$ & $33(73.3)$ & $48(77.4)$ & $122(69.7)$ & 0.192 \\
\hline Instructions on diet and nutrition during perinatal period & $45(69.2)$ & $36(80.0)$ & $52(83.9)$ & $133(76.0)$ & 0.127 \\
\hline Knowledge about health care during pregnancy & $39(60.0)$ & $28(62.2)$ & $47(75.8)$ & $114(65.1)$ & 0.129 \\
\hline Psychological guide during perinatal period & $32(49.2)$ & $21(46.7)$ & $41(66.1)$ & 94 (53.7) & 0.073 \\
\hline Choice of delivery mode & $41(63.1)$ & $31(68.9)$ & $44(71.0)$ & $116(66.3)$ & 0.617 \\
\hline Common symptoms and treatments during pregnancy & $31(47.7)$ & $24(53.3)$ & $38(61.3)$ & $93(53.1)$ & 0.309 \\
\hline Benefit and approaches of breastfeeding & $46(70.8)$ & $28(62.2)$ & $49(79.0)$ & $123(70.3)$ & 0.171 \\
\hline Advices on recovery and contraception after delivering & $28(43.1)$ & $15(33.3)$ & $41(66.1)$ & $84(48.0)$ & 0.002 \\
\hline None of the above & $0(0)$ & $0(0)$ & $1(1.6)$ & $1(0.6)$ & 0.622 \\
\hline \multicolumn{6}{|l|}{ Forms of prenatal education curriculum you participated } \\
\hline Lecture & $64(95.5)$ & $40(88.9)$ & $58(93.5)$ & $162(92.6)$ & 0.445 \\
\hline Group discussion & $33(43.9)$ & $23(51.1)$ & $39(62.9)$ & $95(54.3)$ & 0.257 \\
\hline Video & $37(55.2)$ & $23(51.1)$ & $45(72.6)$ & $105(60.0)$ & 0.043 \\
\hline Demonstration and practice & $36(53.7)$ & $27(60.0)$ & $39(62.9)$ & $102(58.3)$ & 0.563 \\
\hline Promotional materials & $40(59.7)$ & $29(64.4)$ & $44(71.0)$ & $113(64.6)$ & 0.42 \\
\hline \multicolumn{6}{|l|}{ Effect of prenatal education curriculum on mothers of infants } \\
\hline Know more about prenatal examination & $65(100)$ & $41(91.1)$ & $59(95.2)$ & $165(94.3)$ & 0.051 \\
\hline Know more about health care knowledge & $61(93.8)$ & $39(86.7)$ & $58(93.5)$ & $158(90.3)$ & 0.482 \\
\hline Feel more confidence on natural delivery & $62(93.9)$ & $38(86.4)$ & $52(83.9)$ & $152(86.9)$ & 0.273 \\
\hline Know more about the role of doctors and nurses & $57(90.5)$ & $34(75.6)$ & $51(82.3)$ & $142(81.1)$ & 0.045 \\
\hline Feel less fear of delivery & $59(92.2)$ & $38(84.4)$ & $54(87.1)$ & $151(86.3)$ & 0.422 \\
\hline Know more about the benefits and approaches & $60(92.3)$ & $42(95.5)$ & $60(96.8)$ & $162(92.6)$ & 0.742 \\
\hline Know more about how to recovery well after delivery & $53(81.5)$ & $38(84.4)$ & $56(90.3)$ & $147(84.0)$ & 0.777 \\
\hline Can communicate better with the doctors & $55(84.6)$ & $41(91.1)$ & $52(83.9)$ & $148(84.6)$ & 0.295 \\
\hline Feel more trust in doctors & $56(86.2)$ & $39(86.7)$ & $53(85.5)$ & $148(84.6)$ & 0.41 \\
\hline Feel more trust in hospitals & $56(86.2)$ & $38(84.4)$ & $51(82.3)$ & $145(82.9)$ & 0.174 \\
\hline
\end{tabular}

* Fisher's exact test was used

Table 3 Influence of prenatal education curriculum on mothers of infants prenatal examination utilization, delivery mode and recovery status from the latest delivery

\begin{tabular}{llcccc}
\hline Contents & & Prenatal education curriculum & \multirow{2}{*}{$\chi^{2}$} & $P$ \\
\cline { 3 - 4 } & & Participated $n(\%)$ & Not $n(\%)$ & & \\
\hline Prenatal examination utilization & All attended & $99(57.9)$ & $202(48.3)$ & & \\
& Mostly attended & $62(36.3)$ & $180(43.1)$ & & \\
& Rarely attended & $10(5.9)$ & $34(8.1)$ & & \\
& Never attended & $0(0)$ & $2(0.5)$ & 4.778 & 0.029 \\
Delivery mode & Natural delivery & $77(44.0)$ & $198(46.6)$ & & \\
Recovery status & Cesarean section & $98(56.0)$ & $227(53.4)$ & 0.563 & 0.589 \\
& Very well & $36(20.6)$ & $54(12.8)$ & & \\
& Well & $104(59.4)$ & $257(60.9)$ & & \\
& Not sure & $33(18.9)$ & $99(23.5)$ & & \\
& Not well & $2(1.1)$ & $12(2.8)$ & 6.461 & 0.011 \\
\hline
\end{tabular}

throughout the whole perinatal period, ranging from prenatal examination, neonatal screening, breastfeeding, contraception, diet and nutrition instruction, to a psychological guide. Various formats were used, including lectures, group discussion, demonstrations and practice, videos and promotional materials. 
After participating in the prenatal education curriculum, the knowledge and awareness of most mothers on prenatal care, natural delivery and breastfeeding, attitudes towards delivery, hospital and doctors, and ability to communicate with doctors improved. Further, the results showed that prenatal education curriculum participation had a positive influence on mothers' prenatal examination utilization and their recovery status from the delivery. To be specific, mothers who had participated in the prenatal education curriculum had a higher rate of attending prenatal examination, and recovering very well and well from the delivery.

Some previous studies have found that prenatal education can increase awareness and improve the knowledge about risks factors and complications of pregnant women $[7,8]$. Our study found that in addition to the above benefits, prenatal education can also improve women's attitudes towards delivery, hospitals and doctors, and their ability to communicate with doctors. Further we have shown that it can increase the utilization of prenatal examination among pregnant women, and improve their recovery status from the delivery. Therefore, prenatal education should be strongly advocated.

With regard to the provision of prenatal education, a previous study showed that prenatal education provided during routine prenatal visits may be the only health information that pregnant women can receive because of a low attendance rate at formal prenatal education classes [8]. This picture is different for pregnant women in China. In China, due to the heavy workload of gynecologists, who may each need to provide prenatal examination for approximately 50 pregnant women per day; it is impossible for doctors to provide prenatal education to pregnant women during their routine prenatal visits. Therefore, to give pregnant women the necessary knowledge and skills for the perinatal period, a separate prenatal education curriculum is required. Whilst the Chinese NHFPC has recommended that hospitals provide prenatal education curriculum for pregnant women, there is no uniform or standard specification to guide hospitals on providing highquality prenatal education curriculum. Given this, most hospitals in China conduct prenatal education curriculum for pregnant women, but the quality, content and format of the prenatal education curriculum varies in each hospital. To provide high-quality prenatal education for pregnant women, a uniform and standard specification should be made to guide hospitals on how to provide prenatal education curriculum appropriately.

Another problem our study found was that the participation rate of prenatal education curriculum was very low. Without a high participation rate, prenatal education curriculum cannot maximize its potential to improve the overall level of maternal and child health. Accordingly, increasing the participation rate of prenatal education curriculum is crucial. This study found that the most important reasons for pregnant women for not participating were lack of time and access to curriculum in their hospitals, or lack of awareness that their hospitals provided the curriculum. The most important reason for pregnant women participating in the curriculum was their own willingness to learn more knowledge and their doctor's recommendation. In view of this, one way to increase the participation rate is to ask the doctors to publicize the curriculum and highly encourage and advise pregnant women to participate in the curriculum during their routine prenatal visits. A second way is to conduct prenatal education curriculum at a convenient time for pregnant women, so that they are able to participate in the curriculum. A third way is to minimize the length of the curriculum and emphasize the course content, so that it can be delivered to pregnant women in a time-effective manner. With an increased participation rate, more pregnant women can gain benefits from the high-quality prenatal education curriculum provided by hospitals.

There are no studies focusing on the participation in, and implementation of, the prenatal education curriculum provided by hospitals, and its effect on prenatal examination utilization, delivery mode and recovery status either in China. With regard to the implementation of the prenatal education curriculum in different level hospitals, the results showed that only information on "advices on recovery and contraception after delivering" and "video" forms provided by prenatal education curriculum had significant difference. It is suggested that the requirement of the National Basic Public Health Service Specification 2011, issued by the Chinese NHFPC, was well implemented in each level hospitals. On the other hand, the professional advisory skill and the facilities should be more improved in city and county hospitals.

The results of the present study are encouraging and constructive in terms of improving maternal and child health through providing prenatal education curriculum to pregnant women, but the study has a number of limitations. One limitation is that we used a self-report questionnaire as the research tool, so the responses of surveyed mothers may reflect recall error [11]. We have tried to minimize the recall error by selecting mothers who gave birth within 1 year to participate in our study. Another limitation is that we only selected one province as our study area. To get a more comprehensive understanding of the effect of prenatal education curriculum provided by hospitals in China, we need to scale-up our study to more provinces. Thirdly, because of cross-sectional nature of the data, most responses were evaluated retrospectively and recall bias was therefore unavoidable, and finally prospective cohort study should be considered and designed in our future research. 


\section{Conclusion}

Prenatal education has a variety of benefits for women and their babies during the perinatal period. Prenatal education is indispensable for the improvement of the maternal and child health, and thus should be advocated. In China, a standard and convenient specification prenatal education curriculum provided by hospitals and their doctors is appropriated for providing prenatal education to pregnant women.

Acknowledgments The study was funded by a grant from the UNFPA (CHN7U204). The authors would like to thank the participating investigators from Hunan Province for their contribution to data collection. The authors would also like to thank all respondents who enrolled in our study.

\section{Compliance with ethical standard}

Conflict of interest The authors declare that they have no conflict of interest.

\section{References}

1. Mottl-Santiago J, Fox CS, Pecci CC, Iverson R. Multidisciplinary collaborative development of a plain-language prenatal education book. J Midwifery Women's Health. 2013;58(3):271-7.
2. Hawley NL, Brown C, Nu'usolia O, Ah-Ching J, MuasauHoward B, McGarvey ST. Barriers to adequate prenatal care utilization in American Samoa. Matern Child Health J. 2014;18(10):2284-92.

3. Gage A. Barriers to the utilization of maternal health care in rural Mali. Soc Sci Med. 2007;65:1666-82.

4. Halim N, Bohara A, Ruan X. Healthy mothers, healthy children: does maternal demand for antenatal care matter for child health in Nepal? Health Policy Plan. 2010;26:242-56.

5. Guliani H, Sepehri A, Serieux J. Determinants of prenatal care use: evidence from 32 low-income countries across Asia, SubSaharan Africa and Latin America. Health Policy Plan. 2014;29(5):589-602.

6. Hanson L. Prenatal education priorities for perinatal nurses. J Perinat Neonatal Nurs. 2008;22:254-6.

7. Massey Z, Rising SS, Ickovics J. Centering pregnancy group prenatal care: promoting relationship-centered care. J Obstet Gynecol Neonatal Nurs. 2006;35(2):286-94.

8. Ustunsoz A, Senel N, Pollock CA. Comparison of prenatal education delivered by nurses in Ankara (Turkey) and New Orleans (USA). J Clin Nurs. 2010;20:1133-40.

9. Ye D, Li M, Xie D, Zhao L, Wu N, et al. Study on the factors related to cesarean section(CS) in Hu-nan Province by two-level models analysis. Chin J Health Stat. 2010;27:341-4.

10. National Health and Family Planning Commission of the People's Republic of China. National basic public health services project (2011). www.gov.cn/gzdt/att/att/site1/20110524/001e3741 a2cc0f45abb001.doc. Accessed 24 May 2011

11. Osowski CP, Fjellstrom C, Olsson U. Agreement between child and parent reports of 10- to 12-year-old children's meal pattern and intake of snack foods. J Hum Nutr Diet. 2012;25:50-8. 